\title{
Transpalpebral tonometry: reliability and comparison with Goldmann applanation tonometry and palpation in healthy volunteers
}

\author{
A Troost, S H Yun, K Specht, F Krummenauer, O Schwenn
}

Br J Ophthalmol 2005;89:280-283. doi: 10.1136/bjo.2004.050211

Background/aim: TGDc-01 is a new, portable, transpalpebral tonometry device. The aim of this study was to evaluate the reproducibility of this method, including intraobserver and interobserver deviations, and to compare the results with those of Goldmann applanation tonometry and palpation of intraocular pressure (IOP).

Methods: A total of 40 eyes of 20 healthy volunteers were included. Two independent parallel observers (1 and 2) performed three replicate measurements per eye, respectively, both using TGDc01 tonometry, one observer (3) performed three replicate measurements using Goldmann applanation tonometry, and one observer (4) measured the IOP via palpation. Intraindividual deviations within and between both observers using TGDc-01 tonometry and between all three methods were investigated about clinically relevance by comparison of medians and quartiles, statistically significance by pairwise sign tests. Comparisons between observers and methods were based on averaged IOP values of the three individual measurements for each observer and each patient. Intraobserver deviations were analysed by means of Friedman tests.

Results: Observers 1 and 2 showed a statistically significant intraobserver deviation when using TGCc-01 (Friedman $p=0.007$ for observer 1 and $p=0.002$ for observer 2). There was no statistically significant interobserver deviation between observers 1 and 2 (sign test $p=0.200$ ); however, in $45 \%$ of the eyes interobserver deviations were larger than plus or minus $3 \mathrm{~mm} \mathrm{Hg}$. The median intraindividual deviation between TGDc-01 and Goldmann (TGDc-01 minus Goldmann) was $0 \mathrm{~mm} \mathrm{Hg}$ (interquartile range -1 ; $2 \mathrm{~mm} \mathrm{Hg}$; sign test $\mathrm{p}=0.522$ ); but deviations were larger than plus or minus $3 \mathrm{~mm} \mathrm{Hg}$ for $38 \%$ of the 40 eyes. Median intraindividual deviation between TGDc-01 and palpation (TGDc-01 minus palpation) was $-2 \mathrm{~mm} \mathrm{Hg}$ (interquartile range $-4 ; 1 \mathrm{~mm} \mathrm{Hg}$; sign test $p=0.018$ ), but deviations were larger than plus or minus $3 \mathrm{mmHg}$ for $43 \%$ of eyes. Median intraindividual deviation between Goldmann and palpation (palpation minus Goldmann) was $2 \mathrm{~mm} \mathrm{Hg}$ (interquartile range $-2 ; 4 \mathrm{~mm} \mathrm{Hg} ; \mathrm{p}=0.429$ ), but deviations were larger than plus or minus $3 \mathrm{~mm} \mathrm{Hg}$ in $48 \%$ of the eyes. Conclusion: Interobserver deviations using TGDc-01 tonometry and intraindividual deviations between TGDc-01 tonometry, Goldmann applanation tonometry, and palpation of IOP were found to be clinically relevant. Thus, according to our results TGDc-01 could not be established as a substitute or diagnostic alternative method for Goldmann applanation tonometry. But as deviations between TGDc01 and Goldmann turned out smaller than between palpation of IOP and Goldmann, TGDc-01 seems to provide a better choice for tonometry in patients, in whom Goldmann applanation tonometry is not possible.

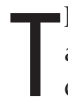
he TGDc-01 (Rjazan State Instrument Making, Russia) is a new, portable, transpalpebral tonometry device, which evaluates the intraocular pressure (IOP) without using invasive procedures. Thus, corneal rigidity and pathology may affect the IOP less compared to Goldmann applanation tonometry.

The aim of this study was to evaluate the reproducibility of this method, including intraobserver and interobserver deviations, and to compare the results with those of Goldmann applanation tonometry and palpation of IOP.

\section{PATIENTS AND METHODS \\ Patients}

The total of 40 eyes of 20 healthy volunteers were included after the subjects had given written informed consent. Patients with upper eyelid and ocular pathologies were not included in this study. Twelve subjects were female and eight were male. Mean age was 24 years (range 22-26 years). All subjects had a visual acuity of $20 / 25$ or better. Mean refraction error was $-1.75 \mathrm{D}$ for the sphere (range $-5.25-$ $2.5 \mathrm{D})$ and $-0.5 \mathrm{D}$ for the cylinder (range $-1.25-0 \mathrm{D})$.

\section{Methods}

The TGDc-01 is a portable, transpalpebral tonometry device (fig 1). The measurement principle is based on determining the acceleration of a free falling rod with a definite weight and its interaction with the elastic eye surface through the eyelid. All measurements were performed on sitting patients, who reclined their head with the glance line being oriented approximately at an angle of $45^{\circ}$. The observer was positioned behind each patient, he pulled up the upper eye lid with his free hand, so that the lid margin touched the limb, and placed the tonometer's tip perpendicular onto the cartilaginous part of the eyelid adjacent and parallel to the lid margin. When the tip of the tonometer touched the eyelid, the measurement (falling of the rod) was automatically started.

Goldmann applanation tonometry was performed after instillation of Thilorbin (Alcon Laboratories Inc, USA) eye drops and positioning the scale of the tonometer on $10 \mathrm{~mm} \mathrm{Hg}$.

Palpation of the IOP was performed by gently pressing the fingertips of both index fingers onto the upper part of the bulbus through the eyelid.

\section{Study design}

On each eye three replicate measurements were taken using the TGDc-01 tonometer by each of two different observers (observers 1 and 2), three replicate Goldmann applanation measurements by one further observer (observer 3), and a fourth observer (observer 4) evaluated the IOP via palpation of the eyes without replicate measurements. The observer sequence was randomised for each patient. Each observer was masked regarding the results of the other observers. 


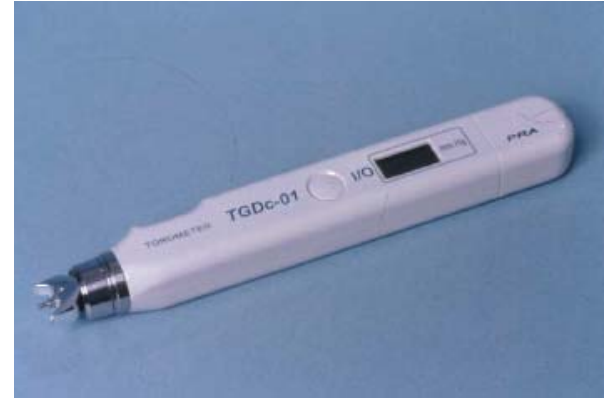

Figure 1 Transpalpebral TGDc-01 tonometer (Rjazan State Instrument Making, Russia).

Ethical permission for the study was granted by the ethics committee of the Landesärztekammer Rheinland-Pfalz, Germany, on 24 March 2003.

\section{Statistical analysis}

All statistical analyses were performed using SPSS 10.0 for Windows. Intraindividual deviations within and between both observers using TGDc-01 tonometry and between all three methods were described by medians and quartiles (graphically by box whisker plots, accordingly), where a clinically relevant deviation was defined as an intraindividual measurement difference of more than plus or minus $3 \mathrm{~mm} \mathrm{Hg}$ before the study. For the sake of device comparison, the replicate measurements of each observer were averaged; furthermore the parallel TGDc-01 measurements of observers 1 and 2 were averaged. Measurement device deviations were analysed for statistical significance by means of pairwise sign tests, where p values $<0.05$ indicate locally significant deviations. Intraobserver deviations were analysed using Friedman tests. Agreement analysis between parallel observers and measurement devices was based on BlandAltman diagrams, where the above plus or minus $3 \mathrm{~mm} \mathrm{Hg}$ deviation was used as a characterisation of clinically relevant method disagreement.

\section{RESULTS}

TGDC-0 1: intraobserver and interobserver deviations Observers 1 and 2 showed a statistically significant intraobserver deviation when using TGCc-01 (Friedman $\mathrm{p}=0.007$ for observer $\mathrm{l}$ and $\mathrm{p}=0.002$ for observer 2) where median measurements ranged between $13 \mathrm{~mm} \mathrm{Hg}$ and $14 \mathrm{~mm} \mathrm{Hg}$ for observer $\mathrm{l}$ and between $13 \mathrm{~mm} \mathrm{Hg}$ and $15 \mathrm{~mm} \mathrm{Hg}$ for observer 2 (table 1). There was no statistically significant interobserver deviation between observers 1 and 2 (sign test $\mathrm{p}=0.200$ ); however, in $45 \%$ of the eyes interobserver deviations were larger than plus or minus $3 \mathrm{~mm} \mathrm{Hg}$ (fig 2). There was a slight IOP level dependency: for lower absolute

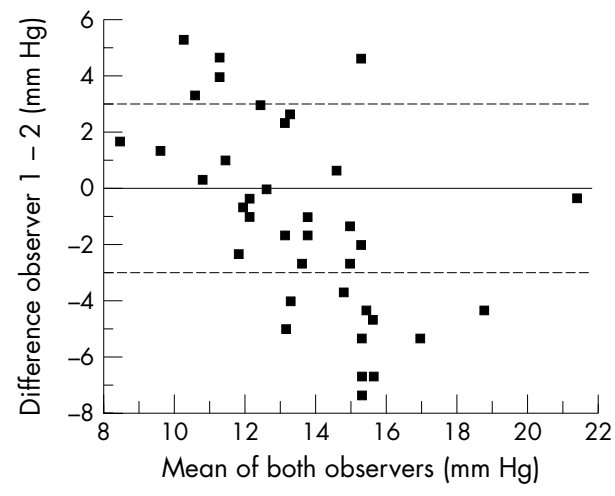

Figure 2 Bland-Altman diagram for the intraindividual IOP measurement deviation between observers 1 and 2 using TGDc-01 tonometry; the dotted horizontal line indicates perfect agreement (difference of $0 \mathrm{~mm} \mathrm{Hg}$ ), the broken lines indicate a clinically relevant difference of plus or minus $3 \mathrm{~mm} \mathrm{Hg}$ (Bland-Altman $95 \%$ agreement limits are represented by the minimum and maximum).

IOP levels the measurement values of observer 1 tended to be larger than those of observer 2 .

\section{Comparison between TGDc-01, Goldmann, and palpation}

The median intraindividual deviation between TGDc-01 tonometry and Goldmann tonometry (TGDc-01 minus Goldmann) was $0 \mathrm{~mm} \mathrm{Hg}$ (interquartile range $-\mathrm{l}$; $2 \mathrm{~mm} \mathrm{Hg}$; sign test $\mathrm{p}=0.522$ ) (table 2, fig 3); but deviations were larger than plus or minus $3 \mathrm{~mm} \mathrm{Hg}$ for $38 \%$ of the 40 eyes (fig 4). Median intraindividual deviation between TGDc$0 \mathrm{l}$ and palpation (TGDc-01 minus palpation) was $-2 \mathrm{~mm} \mathrm{Hg}$ (interquartile range $-4 ; 1 \mathrm{~mm} \mathrm{Hg}$; sign test $\mathrm{p}=0.018$ ) (table 2, fig 3), but deviations were larger than plus or minus $3 \mathrm{~mm} \mathrm{Hg}$ for $43 \%$ of eyes (fig 5 ). Median intraindividual deviation between Goldmann and palpation (palpation minus Goldmann) was $2 \mathrm{~mm} \mathrm{Hg}$ (interquartile range $-2 ; 4 \mathrm{~mm} \mathrm{Hg} ; \mathrm{p}=0.429$ ) (table 2, fig 3), but deviations were larger than plus or minus $3 \mathrm{~mm} \mathrm{Hg}$ in $48 \%$ of the eyes (fig 6).

\section{DISCUSSION}

Many new tonometer have been introduced in recent years. They have all been compared with the Goldmann applanation tonometer, which has been considered the gold standard. ${ }^{1-3}$ When comparing tonometry devices randomisation of methods is necessary because each subsequent measurement lowers the IOP by $0.1-0.4 \mathrm{~mm} \mathrm{Hg.}{ }^{45} \mathrm{~A}$ new tonometry method needs to be accurate and reproducible.

In our study the median intraindividual deviation between TGDc-01 and Goldmann was $0 \mathrm{~mm} \mathrm{Hg}$ (interquartile range $-1 ; 2 \mathrm{~mm} \mathrm{Hg}$ ). Lösch et al demonstrated a mean intraindividual deviation of $3.6 \mathrm{~mm} \mathrm{Hg}$ (SD 4.8) between TGDc-01

Table 1 Descriptive analysis (means, medians, quartiles, minimum, and maximum) of IOP measurements using TGDc-01 tonometry by observers 1 and 2

\begin{tabular}{lllcl}
\hline & $\begin{array}{l}\text { Mean } \\
(\mathbf{m m ~ H g})\end{array}$ & $\begin{array}{l}\text { Median }\left(\mathbf{Q}_{\mathbf{1}} ; \mathbf{Q}_{\mathbf{3}}\right) \\
(\mathbf{m m ~ H g})\end{array}$ & $\begin{array}{l}\text { Minimum } \\
(\mathbf{m m ~ H g})\end{array}$ & $\begin{array}{l}\text { Maximum } \\
(\mathbf{m m ~ H g})\end{array}$ \\
\hline Observer 1 (measurement 1) & 13 & $13(12 ; 14)$ & 8 & 22 \\
Observer 1 (measurement 2) & 14 & $14(12 ; 15)$ & 10 & 22 \\
Observer 1 (measurement 3) & 13 & $13(12 ; 14)$ & 9 & 20 \\
Observer 1 (averaged) & 13 & $13(12 ; 14)$ & 9 & 21 \\
Observer 2 (measurement 1) & 13 & $13(10 ; 16)$ & 7 & 23 \\
Observer 2 (measurement 2) & 15 & $15(11 ; 18)$ & 7 & 22 \\
Observer 2 (measurement 3) & 14 & $14(11 ; 17)$ & 7 & 22 \\
Observer 2 (averaged) & 14 & $14(11 ; 17)$ & 8 & \\
\hline
\end{tabular}




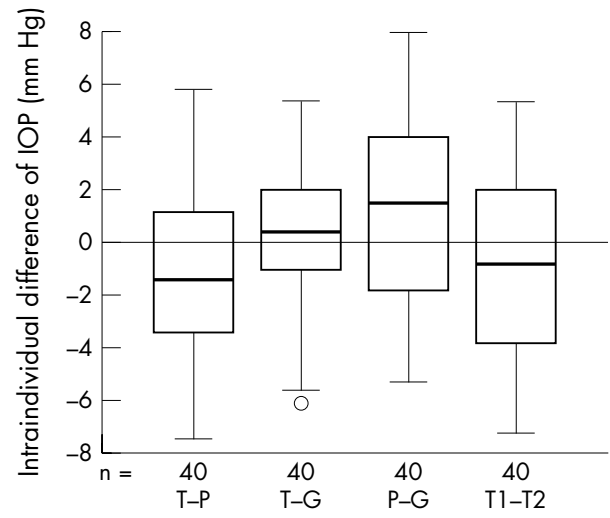

Figure 3 Box and whisker plots of pairwisely intraindividual deviations between measurement devices (based on three replicate measurements of observers 1 and 2 using TGDc-01 tonometry (T1 and T2, respectively) and the latter's average (T), observer 3 using Goldmann tonometry (G) and one measurement by observer 4 palpating the IOP (P); horizontal lines indicate medians and quartiles, whiskers indicate minimum and maximum of intraindividual differences).

and Goldmann in 25 patients with intraocular inflammation, with lower IOP values using TGDc-01. ${ }^{6}$ Rombold et al also found statistically significant lower IOP values with TGDc-01 compared to Goldmann in 100 eyes. ${ }^{7}$ Müller et al, who performed TGDc-01 and Goldmann applanation tonometry in 50 healthy volunteers, did not find statistically significant deviations between both devices: mean IOP with TGDc-01 was $11.2 \mathrm{~mm} \mathrm{Hg}$ (range $7.5-19.5 \mathrm{~mm} \mathrm{Hg}$ ) and with Goldmann $12 \mathrm{~mm} \mathrm{Hg}$ (range 9-19.8). ${ }^{8}$ However, he compared averaged IOP values of a whole population. Thus, interindividual variation of IOP values is to high to detect statistically significant deviations between instruments. Therefore, it is important to evaluate intraindividual device deviations, as we did.

The reason that we did not find a statistically significant deviation between TGDc-01 and Goldmann was not the very good agreement between devices, but the high variability of intraindividual deviations. Deviations between TGDc-01 and Goldmann were more than plus or minus $3 \mathrm{~mm} \mathrm{Hg}$ in $38 \%$ of eyes (fig 4). So, we cannot recommend TGDc-01 tonometry as substitute or alternative method for Goldmann applanation tonometry. But as intraindividual deviations between

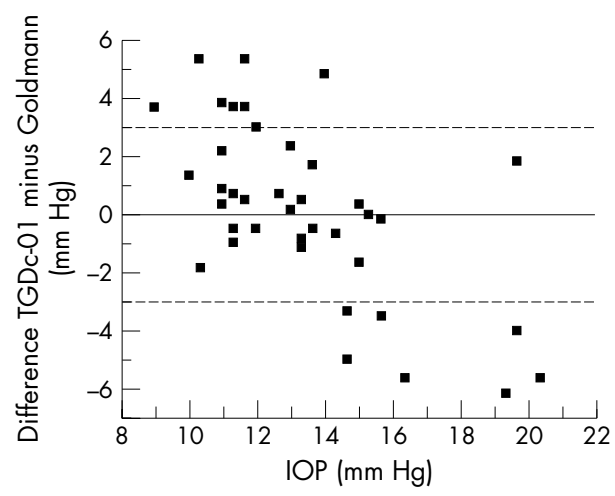

Figure 4 Bland-Altman diagram for the intraindividual IOP measurement deviation between the TGDc-01 tonometry and Goldmann applanation tonometry as a reference; the dotted horizontal line indicates perfect agreement (difference of $0 \mathrm{~mm} \mathrm{Hg}$ ), the broken lines indicate a clinically relevant difference of plus or minus $3 \mathrm{~mm} \mathrm{Hg}$ (BlandAltman $95 \%$ agreement limits are represented by the minimum and maximum).

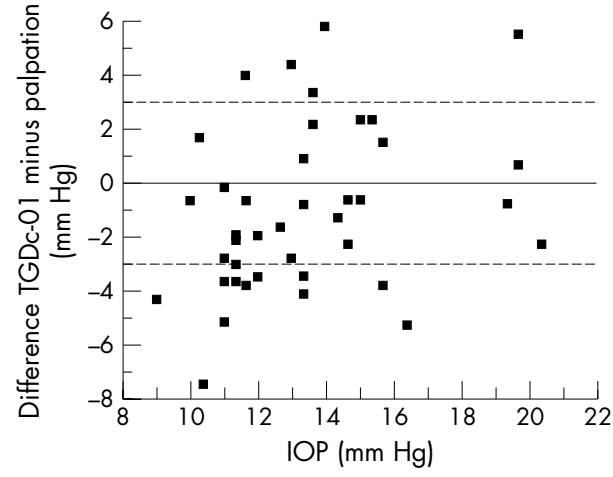

Figure 5 Bland-Altman diagram for the intraindividual IOP measurement deviation between the TGDc-01 tonometry and palpation of IOP as a reference; the dotted horizontal line indicates perfect agreement (difference of $0 \mathrm{~mm} \mathrm{Hg}$ ), the broken lines indicate a clinically relevant difference of plus or minus $3 \mathrm{~mm} \mathrm{Hg}$ (Bland-Altman $95 \%$ agreement limits are represented by the minimum and maximum).

TGDc-01 tonometry and Goldmann tonometry were less compared to those between palpation and Goldmann tonometry (table 2, figs 4 and 6), in cases where Goldmann applanation tonometry is impossible to perform (for example, children without general anaesthesia, corneal pathology) TGDc-01 tonometry seems to be a better choice than palpation, which was the only alternative previously.

Other authors confirm the high variability of intraindividual deviations between TGDc-01 and Goldmann tonometry: Sandner et al demonstrated a deviation of plus or minus $3 \mathrm{~mm} \mathrm{Hg}$ between TGDc-01 and Goldmann tonometry in $12 \%$ of 150 healthy volunteers, range $-11-15 \mathrm{~mm} \mathrm{Hg},{ }^{9}$ and Amm et al in $29.9 \%$ of 80 eyes (40 after keratoplasty). ${ }^{10}$ We are not aware of any other studies comparing TGDc-01 and palpation.

Some authors give correlation coefficients for TGDc-01 and Goldmann between 0.86 and $0.89 .^{9}{ }^{10}$ The correlation coefficient is a measure of the strength of linear association between two variables, which is not the same as a measure of agreement. ${ }^{11}$ Furthermore, the degree of correlation is difficult if not impossible to define, whereas the limits of agreement using Bland-Altman diagrams provide direct information on clinical agreement. ${ }^{12}{ }^{13}$ Worse still, it is possible to obtain a very high correlation while clinical agreement is poor. Correlation analysis is highly sensitive to

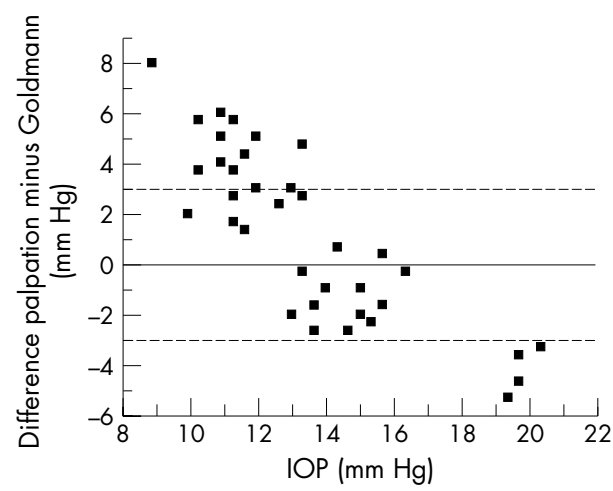

Figure 6 Bland-Altman diagram for the intraindividual IOP measurement deviation between palpation of IOP and Goldmann applanation tonometry as a reference; the dotted horizontal line indicates perfect agreement (difference of $0 \mathrm{~mm} \mathrm{Hg}$ ), the broken lines indicate a clinically relevant difference of plus or minus $3 \mathrm{~mm} \mathrm{Hg}$ (BlandAltman $95 \%$ agreement limits are represented by the minimum and maximum). 
Table 2 Description of the intraindividual comparison (means, medians, quartiles, minimum, and maximum of intraindividual differences) between IOP measurements using TGDc-01 tonometry, Goldmann applanation tonometry, and palpation of IOP (based on averages of three respective replicate measurements for Goldmann applanation tonometry and on averages of two parallel observers' results for TGDc-01 tonometry); sign test $p$ values for pairwise comparisons

\begin{tabular}{|c|c|c|c|c|c|}
\hline & $\begin{array}{l}\text { Mean } \\
(\mathrm{mm} \mathrm{Hg})\end{array}$ & $\begin{array}{l}\text { Median }\left(Q_{1} ; Q_{3}\right) \\
(\mathrm{mm} \mathrm{Hg})\end{array}$ & $\begin{array}{l}\text { Minimum } \\
\text { (mm Hg) }\end{array}$ & $\begin{array}{l}\text { Maximum } \\
(\mathrm{mm} \mathrm{Hg})\end{array}$ & $\begin{array}{l}\text { p Value } \\
\text { (sign test) }\end{array}$ \\
\hline TGDc-01 minus palpation & -1 & $-2(-4 ; 1)$ & -8 & 6 & 0.018 \\
\hline TGDc-01 minus Goldmann & 0 & $0(-1 ; 2)$ & -6 & 5 & 0.522 \\
\hline Palpation minus Goldmann & 1 & $2(-2 ; 4)$ & -5 & 8 & 0.429 \\
\hline TGDc-01 (observer 1-2) & 1 & $-1(-4 ; 2)$ & -7 & 5 & 0.200 \\
\hline
\end{tabular}

the choice of subjects. A high value of $r$ can be obtained because there is large variation between subjects. ${ }^{11}$

Additionally, in our study we showed that for smaller IOP levels TGDc-01 and palpation values exceeded Goldmann values and with higher IOP levels TGDc-01 and palpation values were smaller (figs 4 and 6). So, both tonometry methods, TGDc-01 and palpation, have less power to discriminate between IOP values compared to the gold standard of Goldmann applanation tonometry. As our study population, like all recently published studies, were normotensive patients (range in our study: 9-20 $\mathrm{mm} \mathrm{Hg}$ ), further studies have to be conducted with a wider range of IOP values to confirm this result.

Furthermore the intraobserver and interobserver variability of TGDc-01 was quite high. In our study the intraobserver deviations were statistically significant (Friedman test: observer $1 \mathrm{p}=0.007$, observer $2 \mathrm{p}=0.002$ ). Rombold et al performed five replicate IOP measurements by two different observers in 100 eyes using TGDc-01. Intraobserver variability was $29 \%$ for observer 1 and $8 \%$ for observer 2. Deviations between both observers were statistically significant $(p<0.01){ }^{7}$ Because of the high variability of intraindividual deviations between both observers, we could not show any significant interobserver deviations. But deviations between both observers were more than plus or minus $3 \mathrm{~mm} \mathrm{Hg}$ in $45 \%$ of eyes (fig 2 ), which was clinically relevant. So, TGDc01 demonstrates an observer dependency. And its high intraobserver variability means that we need to perform at least three replicate measurements per eye.

When measuring intraobserver and interobserver variability of new tonometry methods we have to be aware that the gold standard of Goldmann applanation tonometry also demonstrates a rather high variability. Phelbs et al found a deviation between two observers of at least $3 \mathrm{~mm} \mathrm{Hg}$ in $30 \%$ of 420 eyes. $^{14}$ Sudesh et al found a deviation between two observers of at least $3 \mathrm{~mm} \mathrm{Hg}$ in $20 \%$ of 32 eyes. ${ }^{15}$ Some previously identified sources of errors for the Goldmann tonometer are incorrect fluorescein concentration, ${ }^{16}$ illumination, ${ }^{17}$ and the width of the rings.

Possible sources of errors for the TGDc-01 tonometer are:

- the influence of the eyelid, including tarsus and skin, on the elastic eye surface cannot be calculated;

- the elasticity of the sclera is dependent on its thickness and distance to the limbus;

- the perpendicular fall of the rod is not really controlled.

In summary, we demonstrated that interobserver deviations using TGDC-01 tonometry and intraindividual deviations between TGDc-01 tonometry and Goldmann applanation tonometry and between TGDc-01 tonometry and palpation of IOP were clinically relevant. Thus, we cannot recommend the new transpalpebral tonometry device, TGDC-01, as a substitute or alternative method for Goldmann applanation tonometry. But as deviations between TGDc01 tonometry and Goldmann were less than between palpation of IOP and Goldmann, TGDc-01 seems to be a better choice for tonometry in patients with corneal pathology or in awake children than palpation of IOP, at least in normotensive subjects.

\section{Authors' affiliations}

A Troost, K Specht, Department of Ophthalmology, Johannes Gutenberg-University, Langenbeckstrasse 1, 55131 Mainz, Germany

S H Yun, Department of Ophthalmology, Allgemeinkrankenhaus Barmbek (AKB), Rübeukamp 148, 20309 Hamburg, Germany F Krummenauer, Department of Medical Biometry, Epidemiology and Informatics, Johannes Gutenberg-University, Obere Zahlbacher Strasse 69, 55131 Mainz, Germany

O Schwenn, Department of Ophthalmology, Bürgerhospital Frankfurt am Main, Nibelungenallee 37-41, 60318 Frankfurt am Main, Germany

Correspondence to: Dr Annette Troost, Universitäts-Augenklinik, Langenbeckstrasse 1, 55131 Mainz, Germany; Annette.Troost@gmx.de

Accepted for publication 1 August 2004

\section{REFERENCES}

1 Vogel A, Beck S, Schwenn O, et al. Reproduzierbarkeit der Messung von okulärer Pulsamplitude und intraokularem Druck mittels SmartLens. Ophthalmologe $2001 ; 98: 944-9$.

2 Troost R, Vogel A, Beck S, et al. Clinical comparison of two intraocular pressure measurement methods: SmartLens dynamic observing tonography versus Goldmann. Graefes Arch Clin Exp Ophthalmol 2001;239:889-92.

3 lester M, Mermoud A, Achache F, et al. New TonoPen XL: comparison with the Goldmann tonometer. Eye 2001;15:52-8.

4 Moses RA, Liu CH. Repeated applanation tonometry. Am J Ophthalmol 1968:66:89-91.

5 Motolko MA, Feldman F, Hyde M, et al. Sources of variability in the results of applanation tonometry. Can J Ophthalmol 1982;17:93-5.

6 Lösch A, Scheuerle AE, Mackensen F, et al. Measurements of intraocular pressure using TGDc-01 and applanation tonometry. Ophthalmologe 2003; 100(Suppl 1):S67.

7 Rombold F, Thiel M, Neubauer AS, et al. Evaluation of the new mobile TGDc01 tonometer and comparison with the Goldmann applanation tonometry. Ophthalmologe 2003;100(Suppl 1):S68.

8 Müller A, Lang GE, Kampmeier J. Prospective comparison of the tonometer TGDc-01 with the Goldmann applanation tonometry and the non-contact tonometry PT 100. Ophthalmologe 2003; 100(Suppl 1):S67.

9 Sandner D, Pillunat LE, Kostov S. Measurement of the intraocular pressure with the "eyelid tonometer"-TGD c-01 in comparison to applanation and pneumotonometry. Ophthalmologe 2003;100(Suppl 1):S68.

10 Amm M, Hedderich J. Transpalpebral tonometry with a digital tonometer in healthy eyes and after penetrating keratoplasty. Ophthalmologe 2003;100(Suppl 1):S68.

11 Krummenauer F, Genevriere I, Nixdorff U. The biomedical comparison of cardiac imaging methods. Compute Methods Programs Biomed 2000;62:21-34.

12 Bland JM, Alman DG. Statistical methods for assessing agreement between two methods of clinical measurement. Lancet 1986;1:307-10.

13 Altman DG, Bland JM. Measurement in medicine: the analysis of method comparison studies. The Statistitician 1983;32:307-17.

14 Phelps CD, Phelps GK. Measurement of intraocular pressure: a study of its reproducibility. Graefes Arch Klin Exp Ophthalmol 1976;198:39-43.

15 Sudesh S, Moseley J, Thompson JR. Accuracy of Goldmann tonometry in clinical practice. Acta Ophthalmol 1993;71:185-8.

16 Smith R. Applanation tonometry without fluorescence [correspondence] Am J Ophthalmol 1979;87:583.

17 Schmidt T. The clinical application of the Goldmann applanation tonometer Am J Ophtalmol 1960;49:967-78. 